\title{
Calcium deposition as a cause of spoilation of hydrophilic soft contact lenses
}

\author{
M. RUBEN, R. C. TRIPATHI*, AND A. F. WINDER \\ Department of Contact Lens and Prosthetics, Moorfields Eye Hospital, and Department of Pathology, \\ Institute of Ophthalmology, University of London
}

One of the commonest causes of soft lens spoilation is the formation of opacities within the matrix and/or on the surface of the lens. These opacities may develop after varying periods of wear and, at times, after as short as 48 hrs of continuous use. In addition to impairing the optical property of the lens, the opacities can be a cause of lens intolerance. Although aetiological factors, such as degenerative changes in the soft lens material, surface corrosion, bacterial and fungal infection, foreign body implantation, and mucoid encrustation, have been recognized in some cases (Tripathi and Ruben, I972; Milauskas, I972; Kaufman, 1972; Matas, Spencer, and Hayes, 1972; Tragakis, Brown, and Pearce, 1973; Filppi, Pfister, and Hill, r973; Loran, 1973; Dohlman, Boruchoff, and Mobilia, 1973; Sagan and Schwaderer, 1974; Brown, Bloomfield, Pearce, and Tragakis, 1974), the cause of the soft lens opacification remains obscure in most cases.

In many of these lenses, the opacities manifest as discrete spots or as large areas of cloudiness, chalkwhite in appearance. Since similar opacities are known to occur in the cornea and conjunctiva of the eye in a variety of clinical conditions, and histochemical and electron probe microanalytical studies have shown the presence of calcium (Duke-Elder and Leigh, 1965; Fishman and Sunderman, 1966; Berlyne, 1968; Berkow, Fine, and Zimmerman, I968; Doughman, Ingram and Bourne, 1970; Harris, Cohn, Toyofuku, Lonergan, and Galin, 1971), it seemed probable that calcium deposition might be implicated in the spoilation of soft lenses. In this paper, we report the histochemical, electron microscopical, electron probe microanalytical, $x$-ray diffraction, atomic absorption, spectrophotometric, and biochemical analyses of 75 spoilt soft lenses undertaken to identify the physical and chemical characteristics of the opacification, with special reference to the involvement of calcium.

\section{Methods and findings}

Almost all lenses examined in the present series were * Address for reprints: R. C. Tripathi, F.R.C.S., Department of Pathology, Institute of Ophthalmology, Judd St., London WC1H 9 QS of high water content (Sauflon, Bionite, PolyHema), and one was polymethylmethacrylate (PMMA).

\section{(a) Macroscopic appearance of spoilt lenses}

The following features were observed:

Cloudy white appearance

Discrete white spots

White "fungus type" invasion in depth

Coloured spots, usually pigment

Black spots

Homogenous change in colour, either chemically induced by dyes, or secondary to ageing of the polymer.

\section{(b) Surface microscopic appearance of spoilt lenses}

The hydrated lenses in physiological saline were dehydrated by a critical point drying technique, coated with gold palladium, and examined by scanning electron microscopy.

The scanning electron micrographs show examples of surface spoilation, wherein, in addition to lathe

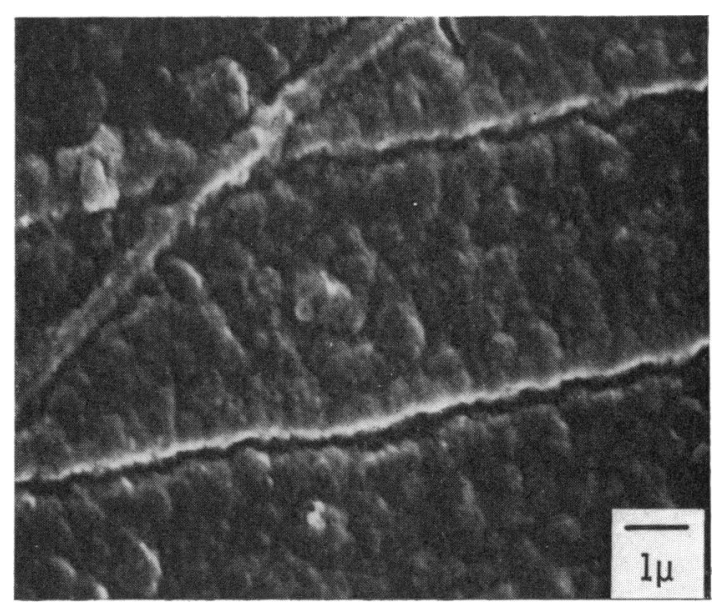

FIG. I Scanning electron micrograph of soft lens

(Sauflon), showing lathe marks in lens surface 


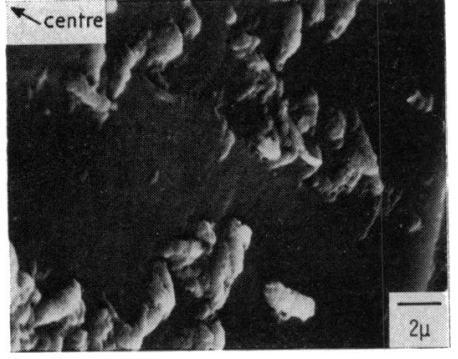

FIG. 2 Scanning electron micrograph of soft lens surface, showing adherent amorphous white deposits

markings, one can note irregularities in troughs of the lathe cuts, pits, and crevices, polymorphous or rounded deposits of variable size, and encrusted plaque deposits (Figs 1, 2, 3).

\section{(c) Light microscopy and histochemistry}

Cryosections stained with haematoxylin and eosin and semi-thin Araldite sections stained with toluidine blue showed widespread small surface irregularities, crevices, and pits on both the outer and inner lens surfaces. The outer part of the lens down to one-third to one-half thickness and including the external surface was heavily impregnated with a dense deposit which tended to be more discrete (round/oval granules $\mathrm{I}-2 \mu \mathrm{m}$ in diameter) and sparser in distribu-

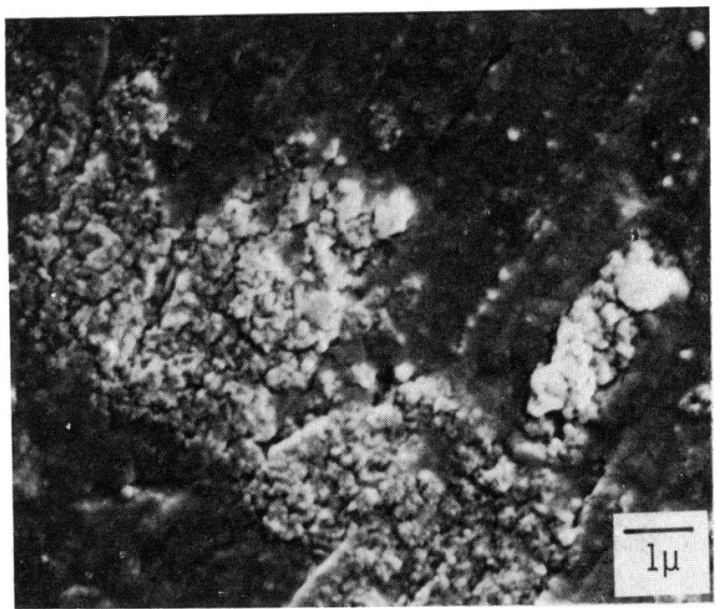

FIG. 3 Scanning electron micrograph, showing encrusted material in soft lens surface. Some lathe marks can also be seen

tion in the deeper lens material (Fig. 4). On the inner aspect of the lens there was a less widespread deposit, orientated in a linear fashion parallel to the lens surface, without much deeper infiltration or discrete granular differentiation. In contrast to the periphery of the lens, the central region was more heavily infiltrated with the deposits.

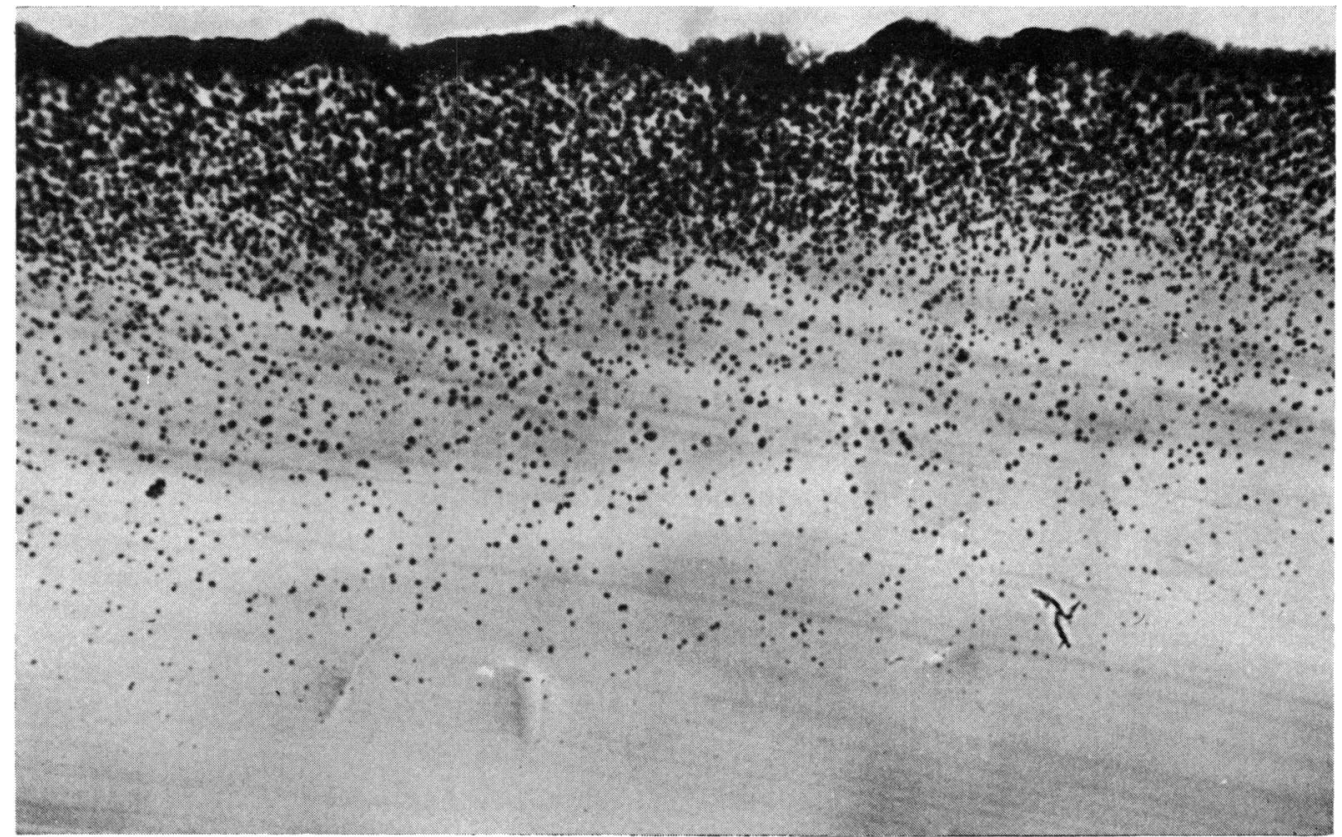

FIG. 4 Light photomicrograph of cross-section of soft lens, showing numerous granular calcareous deposits in outer part of the lens and its surface. Frozen section stained with quinalizarin. $\times 475$ 
The deposits stained black with von Kossa stain, purple with quinalizarin, bright blue with Alcian blue, blue with colloidal iron, and orange-red with alizarin, and gave a negative reaction with periodic acid-Schiff stain. These staining characteristics indicated the presence of calcium.

\section{(d) Electron microscopy}

The lens material cut into blocks of suitable size was fixed with I per cent. osmium tetroxide, dehydrated in ascending grades of ethanol, and finally embedded in Araldite. Thin (6o-10o nm) sections were cut and examined either unstained or after staining with uranyl acetate and lead citrate.

The distribution and differentiation of deposits seen by light microscopy were generally confirmed by. electron microscopy. The deposits appeared to be hard in texture since they were difficult to cut with the glass knives used for ultra-thin sectioning and this presumably caused the scores, knife drags, and stress marks observed (Fig. 5, overleaf).

The linear deposits impregnating the surface of the lens, and the round/oval deposits infiltrating the outer lens area were extremely electron dense. At higher magnification both deposits were seen to consist of a variable proportion of granuloamorphous material and fine needle-like forms, the latter being more abundant in the discrete round/oval deposits (Fig. $6 a)$ and the former in sections stained with uranyl acetate and lead citrate (Fig. $6 b$ ).

\section{(e) Electron-probe x-ray microanalysis (EMMA)}

Sections (120-150 nm thick) cut from the epoxy-resin blocks previously prepared for conventional electron microscopy were mounted on copper grids. The unstained material was examined with an AEI EMMA 4 instrument having the combined features of transmission microscopy and electron probe $x$-ray microanalysis.

Analysis of the lens matrix free of deposits showed the presence of silicon, sulphur, and chloride (Fig. 7a). Analysis of the electron dense deposits, in addition to the above elements, showed the presence of calcium and phosphorus (Fig. $7 b$ ). By subtracting one trace from the other, the analysis of the deposits alone can be obtained (Fig. 7c). The corrected integrated data suggested that the deposits contained calcium and phosphorus in a ratio of approximately $1.5: I$, consistent with their composition as a calcium triphosphate, $\mathrm{Ca}_{3}\left(\mathrm{PO}_{4}\right)_{2}$.

\section{(f) $X$-ray diffraction and atomic absorption analysis}

Two lenses were examined by $x$-ray diffraction analysis, which confirmed that different forms of deposit contributed to the major opacities present.
A yellowish scaly surface deposit contained various elements including phosphorus and calcium, and in one case silicon dioxide, but this surface material was amorphous and a defined mineral structure was not present. A second, more specific deposit, was internal to the lens, and diffraction patterns were consistent with a moderately crystalline carbonate/apatite structure including calcium. A third, markedly opaque, lens was also examined by atomic absorption spectrophotometry, and calcium and silicon were present at trace levels only.

\section{(g) Direct determination of lens calcium}

33 spoilt lenses were examined, obtained from 27 patients with a variety of presenting conditions: thirty lenses were made of Sauflon, two of PolyHema, one of PMMA, and one of Bionite. The collected data are shown in the Table (p. 147).

Each lens was ashed in a closed crucible, the deposit was extracted with hydrochloric acid, and calcium was determined in the supernatant after incomplete neutralization and centrifugation by an automated fluorimetric procedure involving the production of a fluorescein complex (Hill, ig65). Values were calculated from a standard curve prepared at the same time, the reagent blanks and occasionally values for hydrated unpolished and unfitted lens discs were also determined.

The spoilt lenses were chosen at random and initially were submitted for analysis only if badly affected. Calcium values recorded ranged from $0-250 \mu \mathrm{g}$. per lens with values of $0-12 \mu \mathrm{g}$. for the control discs, and there was a broad correlation between the degree of spoilation and the amount of calcium found. Of the 33 spoiled lenses, sixteen gave values above the control level of $12 \mu \mathrm{g}$. and of these eight contained more than $50 \mu \mathrm{g}$. calcium, including three above $200 \mu \mathrm{g}$. and two in the 100-200 $\mu \mathrm{g}$. range (Table). The two Polyнema lenses from different patients contained 62 and $232 \mu \mathrm{g}$. calcium, but in general a breakdown of the results did not reveal any obvious correlation between the amount of calcium in the deposit and the type of lens, the duration or conditions of use, or the primary condition requiring treatment.

\section{Discussion}

The present study has shown that calcium is implicated in deposit formation in many cases, and that a broad correlation exists between the amount of calcium present and the degree of opacification. Deposition of calcium occurred not only in high water content soft lenses but also in one lens of low water content analysed in this investigation. Györffy (1972) reported calcium deposits on PMMA corneal lenses as a cause of intolerance. Sibley and Yung (r973) have shown that hydrophilic HEMA materials show 


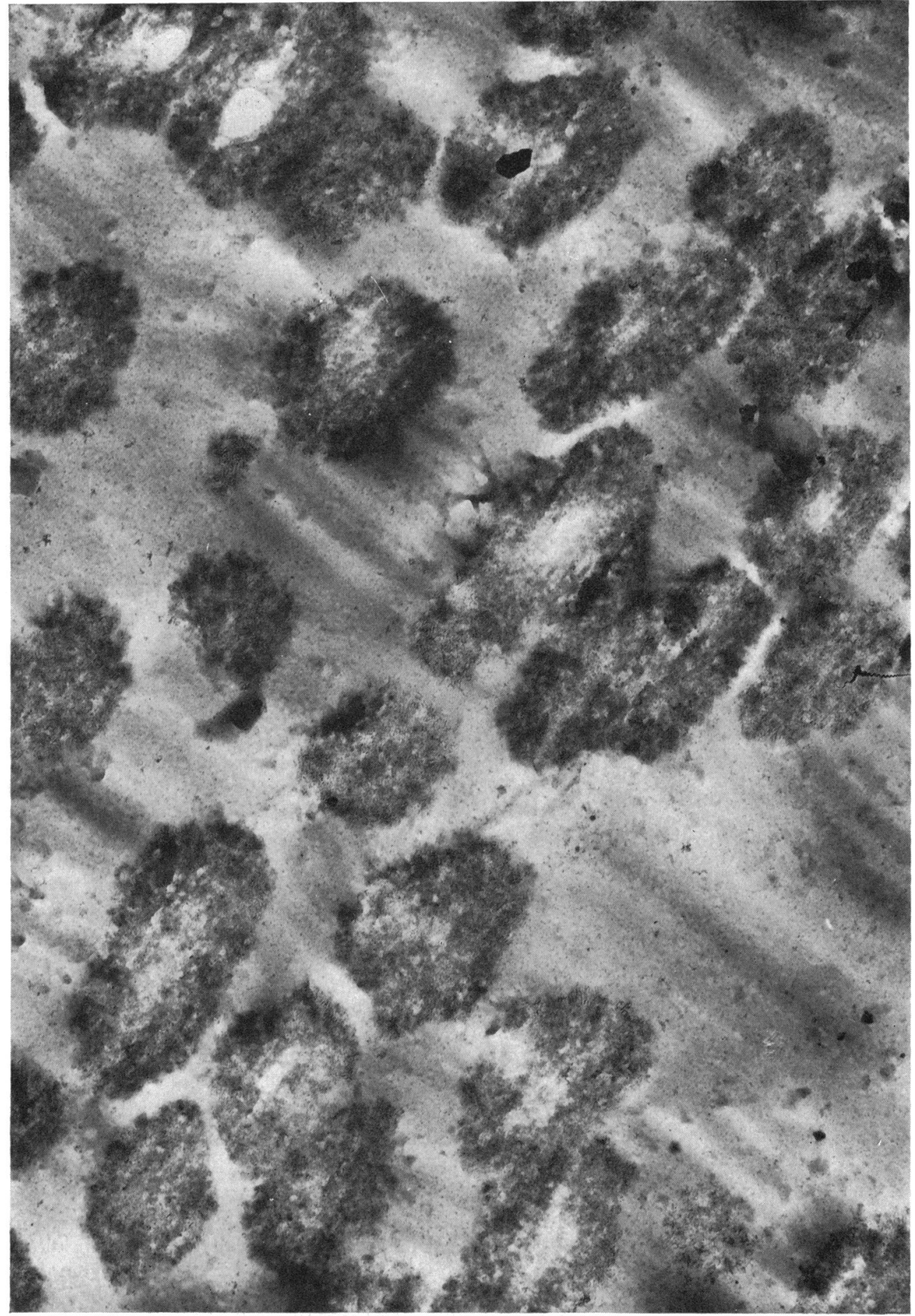

FIG. 5 Electron micrograph of oval/circular electron dense deposits in anterior lens substance. Note knife drags distorting organization of deposits. Uranyl acetate and lead citrate. $\times 20,000$ 


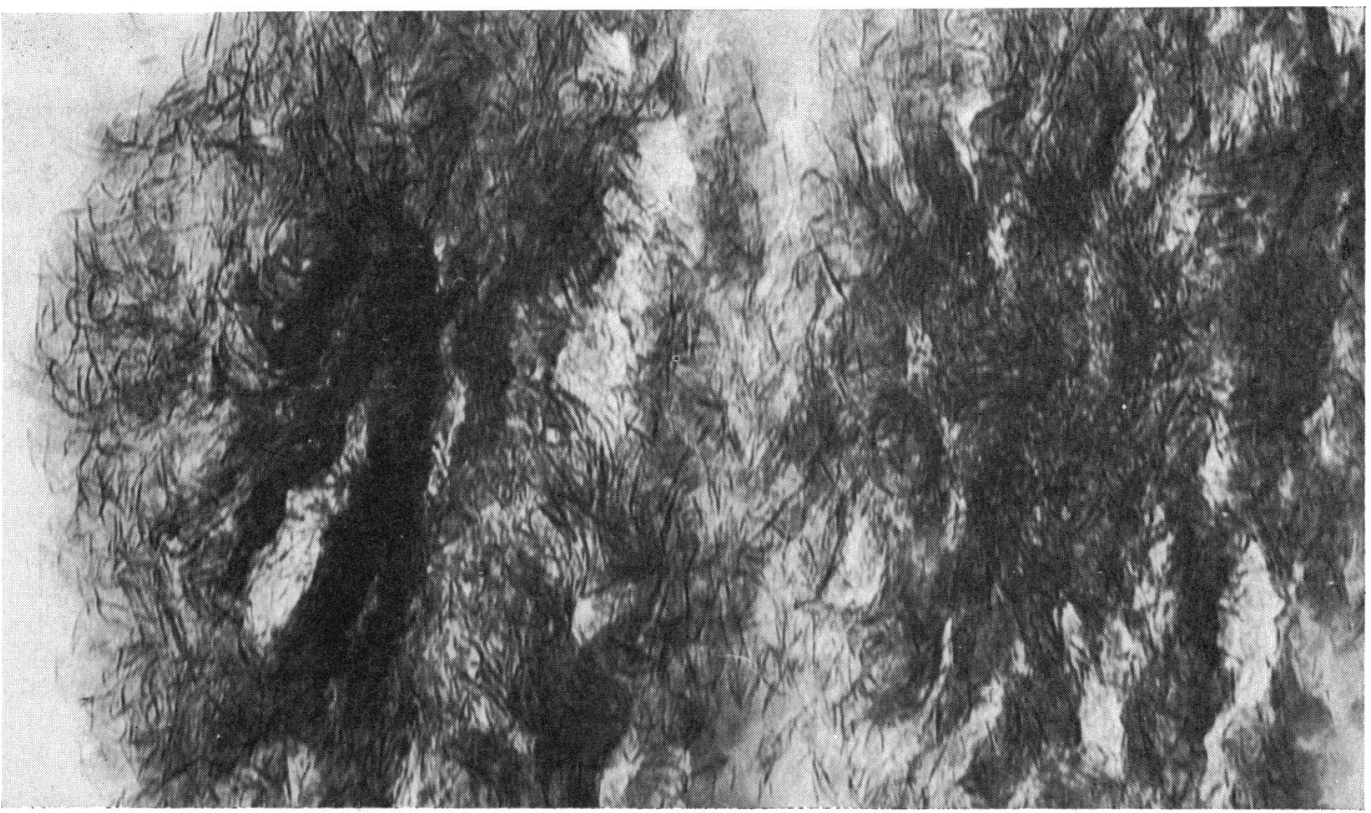

FI G. 6(a) Electron micrograph of discrete deposits in lens substance as seen in an unstained preparation. Note predominance of needle-like structures associated with electron lucent matrix; in unstained preparation surrounding lens material appears electron lucent. $\times 70,000$

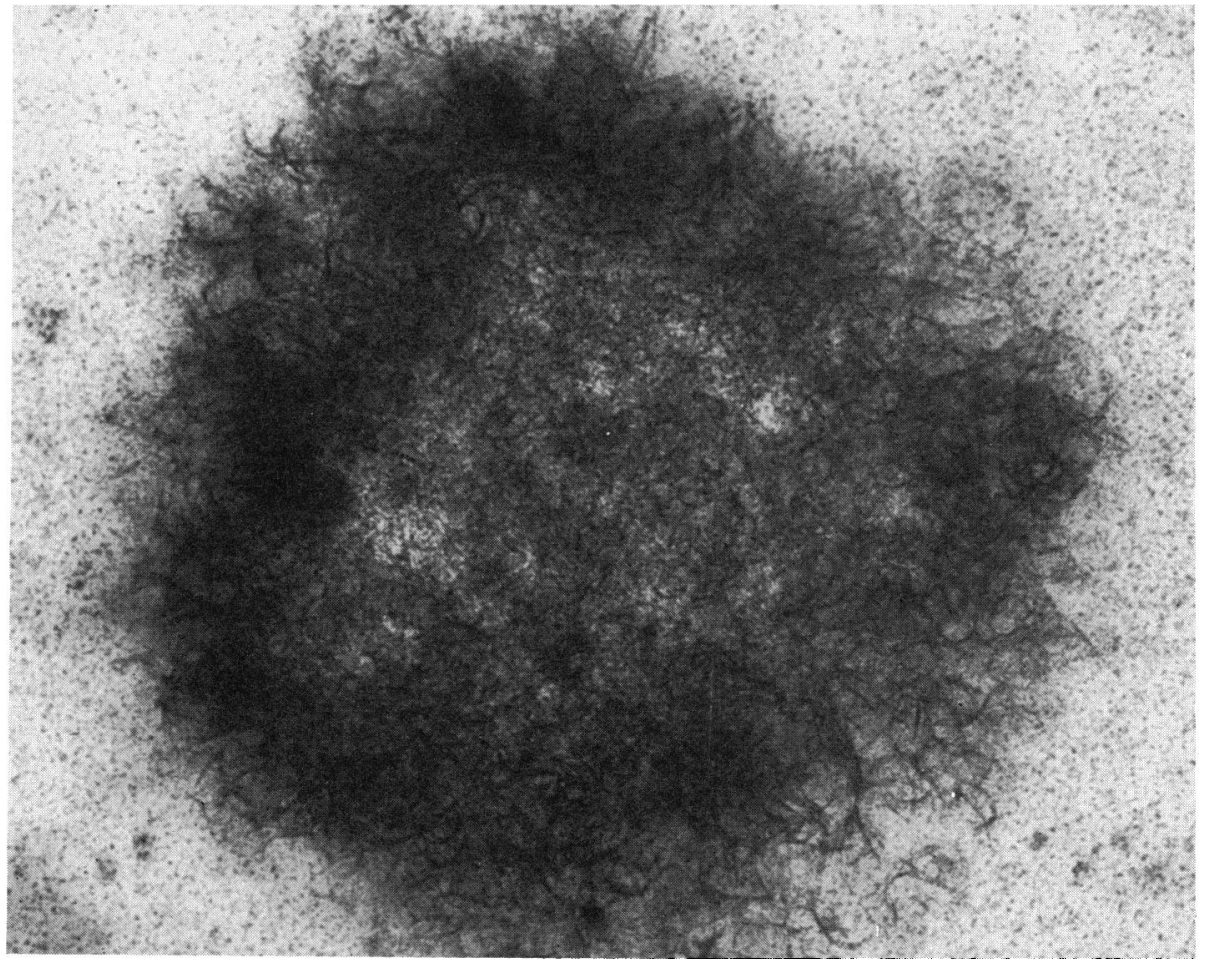

FI G. 6(b) After staining of sections with uranyl acetate and lead citrate, deposits in lens substance exhibit increased electron density of the matrix, associated with needle-like structures. The particulate appearance of the surrounding lens material probably corresponds to the molecular arrangement of the co-polymer that has been rendered apparent because of the staining reaction. Electron micrograph. $\times 75,000$ 
selective molecular absorption and this property is associated with a positive ion charge. Therefore, the deposition of calcium salts or conglomerates may follow either pattern or both. The transmission electron microscopical, electron-probe microanalytical, and histochemical data suggest that, in at least some cases, relatively specific microcrystalline deposits are formed with selective patterns of distribution; these observations carry the implication that specific and definable factors may be involved in calcium deposition. It is known that soft lenses can be penetrated by high molecular weight material and that entry of material into the lens may be facilitated by the cracks and crevices which develop on the surface of the lens with use (Tripathi and Ruben, 1972).

In considering the source of deposited calcium in the soft lenses, apparently two main possibilities must be borne in mind, namely the tears and the keratoconjunctival tissue. If there is an excess calcium level either in freshly secreted tears or liberated into the tears from pathological tissue, it is possible for abnormal deposits of the calcium salt to build up on or in the lens matrix. From the study of the present series, however, there appears to be no obvious direct correlation between the disease conditions and calcium deposition, although marked spoilation occurred in those instances associated with tissue necrosis. Even if the relative proportion of calcium is not raised, its total value could be greater owing to the increased volume of tear secretion since the soft lens provides a mild stimulus for tear production (Tripathi, 1975). This consideration is less applicable, however, in cases of 'dry' eye syndromes, but in such instances the factor of tissue necrosis, tear substitutes, and other medicaments applied to the eye, may have a significant part to play.

The influence of factors such as enzymes, oxygen tension, temperature gradient, $\mathrm{pH}$, drugs, and other

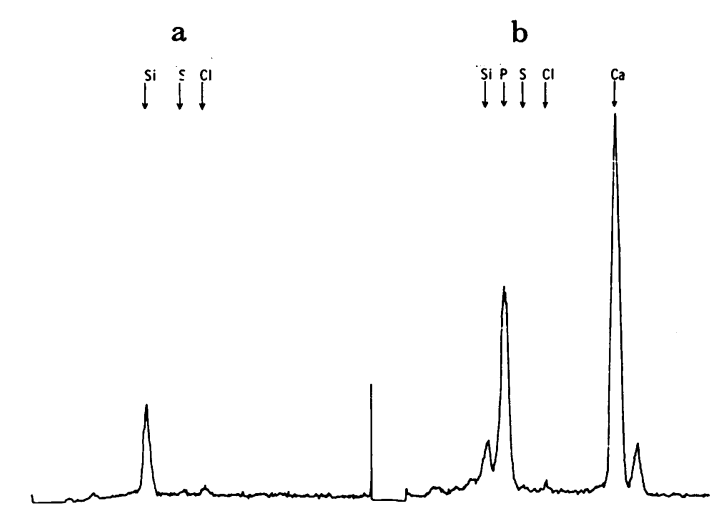

chemicals which come into contact with soft lenses during their use, in favouring deposition of calcium, remains at present a matter for speculation. It would seem significant to note that soft lenses can show a higher than normal lactic acid concentration when worn for continuous periods (Ruben and Carruthers, 1972) and in these cases the $\mathrm{pH}$ of the lens may determine the deposition of calcium (as a crystalline salt or as conglomerate). In explaining the mode of calcium deposition in the cornea, it has been suggested that the decreased $\mathrm{pCO}_{2}$ (due to conjunctival exposure) would be associated with a rise in $\mathrm{pH}$ and that in the presence of elevated levels of calcium and phosphorus, as for example, in uraemic patients, calcium phosphate might precipitate (Cogan, Albright, and Bartter, 1948). On the other hand, the palpebral fissure area is exposed and is an area subject to surface drying, thus leading to a greater concentration of calcium salts and their precipitations. Correlation between calcium deposition and analyses of the patients tears and of drops of proprietary solutions used would obviously be informative in defining specific factors, but in the present study these factors have not been thoroughly analysed.

Calcium deposition is a major factor in lens spoilation with opacification but there must be other causes since biochemical analysis of many randomly selected opaque lenses did not reveal higher than normal values for calcium concentration. The presence of silicon in the lenses examined by $x$-ray diffraction and atomic absorption analyses is unexplained; they may be casual contaminants or silicone derivatives which are sometimes used as fillers in the lens polishing process, and modification of this filler or its attachment may contribute to lens spoilation. This suggestion is, however, merely speculative, and electron probe microanalysis of neighbouring clear and opaque areas of the spoiled Sauflon lens

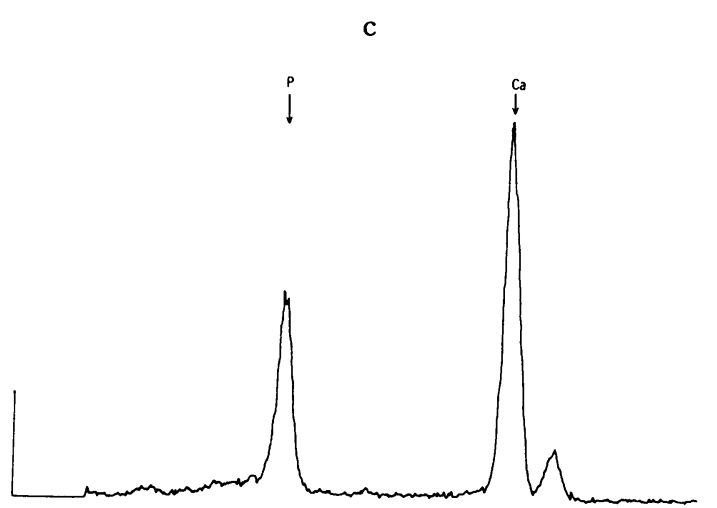

FIG. 7 Traces obtained from electron microprobe $\mathrm{x}$-ray analysis

(a) Elements present in matrix (Si, S, Cl)

(b) Elements present in deposit ( $\mathrm{Si}, \mathrm{P}, \mathrm{S}, \mathrm{Cl}, \mathrm{Ca})$

(c) Background subtracted analysis of deposits, showing presence of calcium (Ca) and phosphorus $(P)$ 
Table Calcium deposition in soft lenses: relationship with patient and diagnosis, lens material, and conditions of use

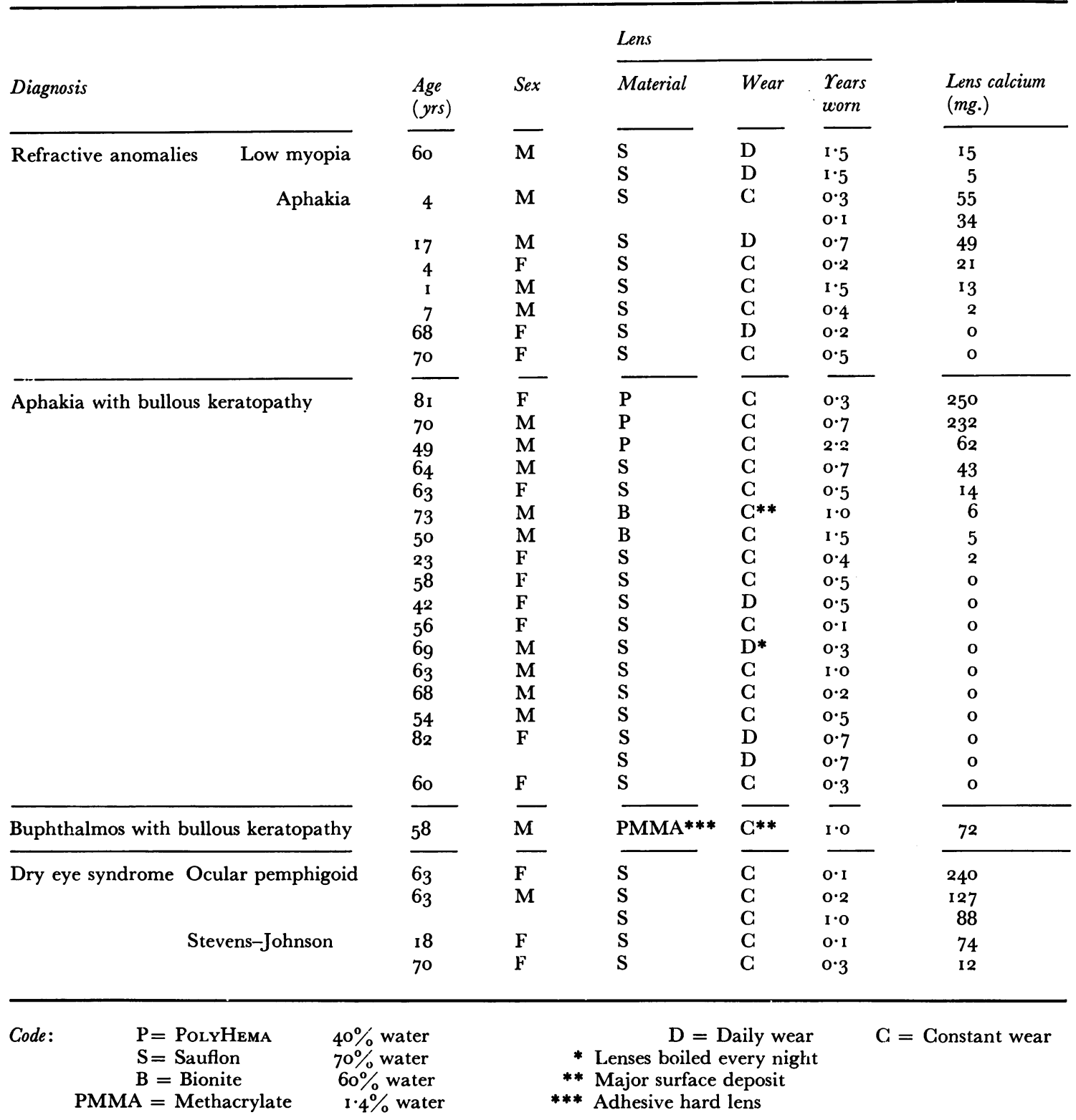

did not reveal any difference in the levels of silicon present.

As far as the lens is concerned, calcium deposition leads to lens spoilation and impairs light transmission. In addition to the physical presence of the deposits, the breaks in surface continuity and reduction of smooth texture, as clearly revealed by scanning electron micrographs, would appear to add to the problem of lens intolerance. There is obviously a need to identify the conditions favouring deposition of calcium in order to prevent or nullify this process. Whilst calcium removal from lenses can be affected by acid solutions and EDTA, this would seem undesirable since salts once deposited and then dissolved away leave pits and irregularities in the surface and/or in the matrix which are also a cause of lens spoilation. The ideal soft hydrophilic contact lens, therefore, will be one which does not permit calcium 
deposition. Improvements may thus involve the use of modified or new lens material and different conditions of use, together with improved assessment of the systemic and ocular syndromes encountered to identify the patient at risk of early lens failure.

\section{Summary}

A series of 75 spoilt soft lenses with opacities (mostly manifesting as discrete spots or as large areas of cloudiness, chalk-white in appearance) were subjected to histochemical, electron microscopical, electron probe $x$-ray microanalytical, $x$-ray diffraction, atomic absorption spectro-photometric, and biochemical analyses. The results showed that in many cases calcium was implicated in the deposit formation and that a broad correlation existed between the amount of calcium present and the degree of opacification. The possible mode of calcium deposition and resulting implications in lens intolerance are discussed. It is advocated that a search should be made for a modified or new soft lens material which does not permit calcium deposition, and that the medical practitioner should strive towards improved assessment of the systemic and ocular conditions which may help in identifying patients at risk of early lens failure.

We wish to thank Dr. William Coombs, of Bausch and Lomb Ltd., for arranging scanning electron microscopy of several lenses from this series; AEI Scientific Apparatus. and Dr. G. W. Lorimer and Mr. G. Cliffe of the Metallurgy Department, Manchester University, for their help in the electron microprobe $x$-ray analysis; Mr. R. K. Harrison and Mr. B. R. Young of the Institute of Geological Sciences, University of London, for carrying out the $x$-ray diffraction studies; Mr. B. R. Frary, of Shandon Southern Instruments Ltd., for assistance with the atomic spectroscopy; Dr. M. Carruthers for performing some of the early calcium analyses; and Mr. A. J. Bron for a case referral.

\section{References}

ABrams, J. D. (ig66) Proc. roy. Soc. Med., 59, 533

Berkow, J. W., fine, B. s., and zimmerman, L. E. (1968) Amer. F. Ophthal., 66, 81 2

BERLYNE, G. M. (1968) Lancet, 2, 366

BROWN, S. I., BlOOMfield, S., PEARCE, D. B., and tragakis, M. (1974) Arch. Ophthal., 91, 275

COGAN, D. G., ALBRIGHT, F., and BARTTER, F. C. (I948) Ibid., 40, 624

DOHLMAN, C. H., BORUCHOFF, A., and MOBILIA, E. F. (I973) Ibid., 90, 367

DOUGHMAN, D. J., INGRAM, M. J., and BOURNE, w. M. (I970) Invest. Ophthal., 9, $47 \mathrm{I}$

DUKE-ELDER, S., and LEIGH, A. G. (1965) "System of Ophthalmology", vol. 3, pt. 2. Kimpton, London

FILPPI, J. A., PFISTER, R. M., and HILL, R. M. (1973) Amer. F. Optom., 50, 553

FISHMAN, R. S., and SUNDERMAN, F. w. (1966) Arch. Ophthal., 75, 367

GYÖRFFY, I. (1972) Contact Lens, 3, pt. 7, p. I9

HARRIs, L. s., GOHN, K., TOYOFUKU, M., LONERGAN, E., and GALIN, M. (197I) Amer. F. Ophthal., 72, i 30

HILL, J. B. (1965) Clin. Chem., I1, I 22

KaUfman, H. E. (1972) In Milauskas (1972) Discussion

LORAN, D. F. C. (I973) Contact Lens, 4, pt 4, p. 3

milauskas, A. T. (1972) Trans. Amer. Acad. Ophthal. Otolaryng., 76, $5 \mathrm{I} 5$

MAATS, B. R., SPENCER, W. H., and hAYES, T. L. (I972) Arch. Ophthal., 88, 287

RUBEN, M., and CARRUTHERS, M. (1972) Contact Lens, 4, pt I, p. 8

SAGAN, w., and schwaderer, K. N. (1974) F. Amer. opt. Ass., 45, 266

SIBLEY, M. J., and YUNG, G. (1973) Amer. F. Optom., 50, 710

TRAGAKIS, M., BROWN, S. I., and PEARCE, D. B. (1973) Amer. F. Ophthal., 75, 496

TRIPATHI, R. C. (1975) "Applied physiology and anatomy: tears, cornea, conjunctiva, and ocular adnexa"

in "Contact Lens Practice", ed. M. Ruben, chap. 3. Baillière Tindall, London and RUBEN, M. (1972) Ophthal. Res., 4, 185 